\title{
Radiofrequency Catheter Ablation of Left Free Wall Accessory Pathways: Comparison Between Retrograde and Transeptal Approach
}

\author{
S Hashem ${ }^{1}$, M Hossain ${ }^{1}$, M.A Ali ${ }^{1}$, A K Choudhury ${ }^{1}$, A A Masum ${ }^{2}$ \\ ${ }^{1}$ Depatment of Cardiology, NICVD, Dhaka. ${ }^{2}$ Fuad Al Khatib Hospital, Cox's Bazar.
}

Key Words: Radio frequency ablation, Arrhythmia, Accessory pathway.

\begin{abstract}
Background : This study was conducted to compare the conventional retrograde arterial approach (RAA) with the transeptal approach (TSA) for radiofrequency catheter ablation (RFA) of left accessory pathways (AP).

Materials and Methods: Sixty consecutive patients (44 male; mean age of $35.60 \pm 11.63$ years) with 60 left APs (39 overt and 21 concealed) underwent catheter ablation using the TS method (30 patients) and the RA method (30 patients) in an alternate fashion. The analysis was performed according to the intention-to-treat principle.

Results : The transeptal puncture was successfully performed in 29 patients (96\%). This access allowed primary success in the ablation in all the patients without any complication. When we compared this approach with the RAA there was no difference as regards the primary success $(p=$ 0.103), fluoroscopy time $(p=0.565)$ and total time $(p=0.1917)$. Three patient in the RAA group presented a vascular complication. The TSA allowed shorter ablation times $(p=0.006)$ and smaller number of radiofrequency applications $(p=0.042)$ as compared to the conventional RAA. The patients who had unsuccessful ablation in the first session in each approach underwent with the opposite technique (cross-over), with a final ablation success rate of $100 \%$.

Conclusion: The TS and RA approaches showed similar efficacy and safety for the ablation of left accessory pathways. The TSA allowed shorter ablation times and smaller number of radiofrequency applications. When the techniques were used in a complementary fashion, they increased the final efficacy of the ablation
\end{abstract}

(Cardiovasc. j. 2012; 4(2): 139-147)

\section{Introduction:}

Radiofrequency ablation (RFA) is currently the treatment of choice for most patients with accessory pathway-mediated tachycardia. Left accessory pathways are most commonly found in clinical practice and account for $40 \%-70 \%$ of all Accessory pathway cases referred for catheter ablation. ${ }^{1-4}$ Because of the inherent risks of the approach of the heart's left chambers special technical skills are required for their of the Accessory pathways mapping and ablation.

Two major methods have been described for the approach of Accessory pathways in the mitral ring: the retrograde arterial approach (RAA) which involves a peripheral arterial access, with the manipulation of the catheter in the left ventricle to map the atrioventricuiar ring (in this approach the atrial connection may also be mapped using retrograde catheterization of the LA) and the transeptal approach (TSA) which consists in trespassing the interatrial septum with a special catheter introducer and place the ablation catheter directly in the LA to map preferably the atrial insertion of these Accessory pathways.

The RA approach is the most widely used in most laboratories. However, with the advancements in ablation procedures for atrial arrhythmias, markedly for atrial fibrillation, the transeptal puncture has been incorporated to the routine of electrophysiologists. Retrospective studies have compared these two techniques and reported controversial results, Usually related to the experience of each group. ${ }^{5-13}$

The objective of this study was to prospectively compare the efficacy and safety of these techniques in a consecutive group of patients reffered for catheter ablation of left Accessory pathways.

\section{Study Method:}

Sixtyn consecutive patients were included in this study, among 44 were male, with a mean age of 35.60 \pm 11.63 years (ranging from 25 to 45 years) with 60 left APs (39 overt and 21 concealed) referred for $\mathrm{RF}$ ablation. Only five patients presented structural heart

Address of correspondence: Dr Sabina Hashem, Department of Cardiology, National Institute of cardiovascular Diseases, Dhaka, Bangladesh. 
disease, three patient with rheumatic valvulopathy and two with atrial septal defect.

The exclusion criteria were: children under twelve years of age; presence of multiple accessory pathways, complex congenital cardiomyopathies, patent oval foramen, repaired interatrial communication, peripheral arterial disease or aortic disease, mechanical prosthesis in the aortic or mitral valve position, symptomatic coronary artery disease, catheter ablation prior to the beginning of the study.

After diagnostic confirmation by electrophysiological study (EPS) the patients were systematically assigned the RAA or TSA in an alternate manner according to a data base with no prior knowledge on the part of the operator, thus ensuring the randomization of the sample studied. The unsuccessful cases were assigned for the other technique (cross-over).

The procedure was performed after an 8-hour fast, upon written consent from the patient or other qualified person, and after the interruption of antiarrhythmic drugs for five half-lives and of amiodarone hypochioride for a month. under sedation and analgesia with intravenous midazolam and phentanyl, two 7Fr quadripolar and hexapolar catheters were introduced through a puncture in the right femoral vein and guided by fluoroscopy until they were placed in the high right atrium (RA) and the bundle of His region. Through a puncture in the left femoral vein a 7Fr decapolar, catheter was placed in the coronary sinus; in order to record the ventricular electrogram and the ventricular stimulation a quadripolar catheter was introduced in the right ventricle (RV). A multi-channel data

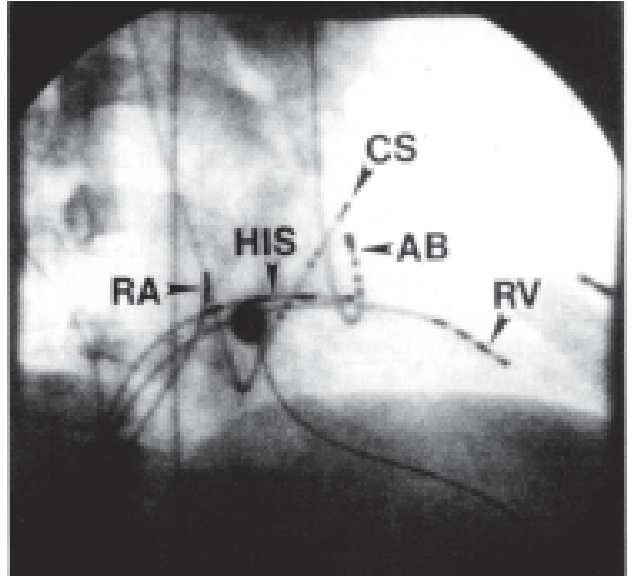

A acquisition system manufactured by Bard Electrophysiology, USA was used for data recording. The system consisted of an amplifier for acquiring and processing surface ECG and intracardiac signals, multi-channel display screens and a computer hard drive to store the ECG and intracardiac signals. The amplifier filtered the intracardiac signals between 30 and $500 \mathrm{~Hz}$. For most studies 4 surface and 8 to 10 intracardiac signals were displayed simultaneously. However, the computer stored simultaneous 12 lead ECG and multiple intracardiac signals which could be viewed real time at speeds up to $400 \mathrm{~mm} / \mathrm{sec}$. A physiological cardiac stimulator manufactured by Micropace Ltd, Australia was used during the procedures. It is an electrically isolated unit is capable of delivering constant current pacing impulses. A radiofrequency ablation generator manufactured by Boston Scientific, USA, model EPT-1000 XP was used for ablation. This unit is capable of monitoring temperature, power and impedance during radiofrequency current application. It was connected to intracardiac ablation catheter via a junction box and the radiofrequency current was delivered between the catheter tip and an external grounding pad placed on the back of the patient. For the TS approach we used long introducers (Swartz ${ }^{1 \mathrm{M}}$ Guiding Introducers, SL2, St. Jude Medical Company) and Brockenbrough needles.

Rertrograde arterial technique - Guided by fluroscopy, the ablation catheter was introduced through a puncture in the right femoral artery up to the left ventricular cavity, the mapping was started. As part of the routine, intravenous heparine (5000 UI bolus IV followed by 1000 UI per hour)was used for anticoagulation.

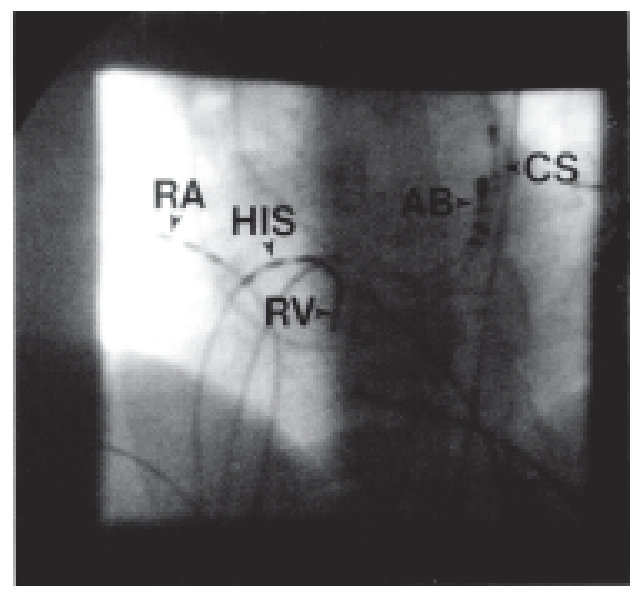

B

Fig.-1: Catheter positions in right anterior oblique (A) and left anterior oblique (B) for ablation of left free wall accessory pathway by the retrograde transaortic approach. 
Transseptal technique- transeptal puncture technique were performed by the electrophysiology team of national Institute of Cardiovascular Diseases and Hospital. Monoplane fluoroscopic projections were used (right anterior oblique $45^{\circ}$ and left anterior oblique $40^{\circ}$ ) in all cases. The transseptal sheath/dilator was placed in the superior vena cava and then the dilator/sheath/ needle combination was withdrawn inferiorly in the left anterior oblique projection until the tip of the dilator "popped" into the fossa ovalis. Positioning was always checked in the right anterior oblique projection to ensure that the tip of the needle/sheath assembly was pointing directly away from the plane of vision and slightly anterior. A test injection of contrast was delivered through the dilator in the left anterior oblique projection to check for "tenting" of the fossa at the intended puncture site. This was typically just below the level of the His recording catheter in the left anterior oblique $40^{\circ}$ projection, and well behind the His catheter electrodes in the right anterior oblique $45^{\circ}$. The needle was then advanced and a second test injection was made following needle advancement to check that the needle had entered the left atrium; left atrial pressure was then recorded. The sheath \& dilator was advanced over the needle into the left atrium. Once the dilator was well into the left atrium, the sheath was advanced over the dilator, but the needle remained just inside the dilator to give support for advancement of the long sheath. The dilator with the needle was then removed. 5000 units of heparin was given intravenously followed by further 2500 units being given if left sided deployment lasted more than one hour. The sheath was connected to a continuous heparinized flush at about 2 to 3 cc per hour to prevent clot formation inside the sheath. Patients remained in the laboratory with transseptal sheath in place for 30 minutes after the procedure for confirmation of success. After successful ablation, transseptal sheaths were withdrawn across the interatrial septum with the ablation catheter tip protruding slightly. This technique was employed to avoid inadvertent displacement of adherent coagulum from the catheter at the sheath tip.

\begin{abstract}
Ablation
Ablations were performed using $4 \mathrm{~mm}$ tip EPT catheter. (EP Technology, Boston scientific)). The ablation catheter was passed through the sheath into the left atrium and positioned on the mitral annulus. Care was always taken to aspirate blood and flush the sheath prior catheter advancement to avoid entrainment of air. Suitable ablation sites were obtained using conventional criteria. The RF application was performed in sinus rythm in overt accessory pathways and with ventricular stimulation in concealed Accessory pathways. We interrupted the Radiofrequency application in the
\end{abstract}

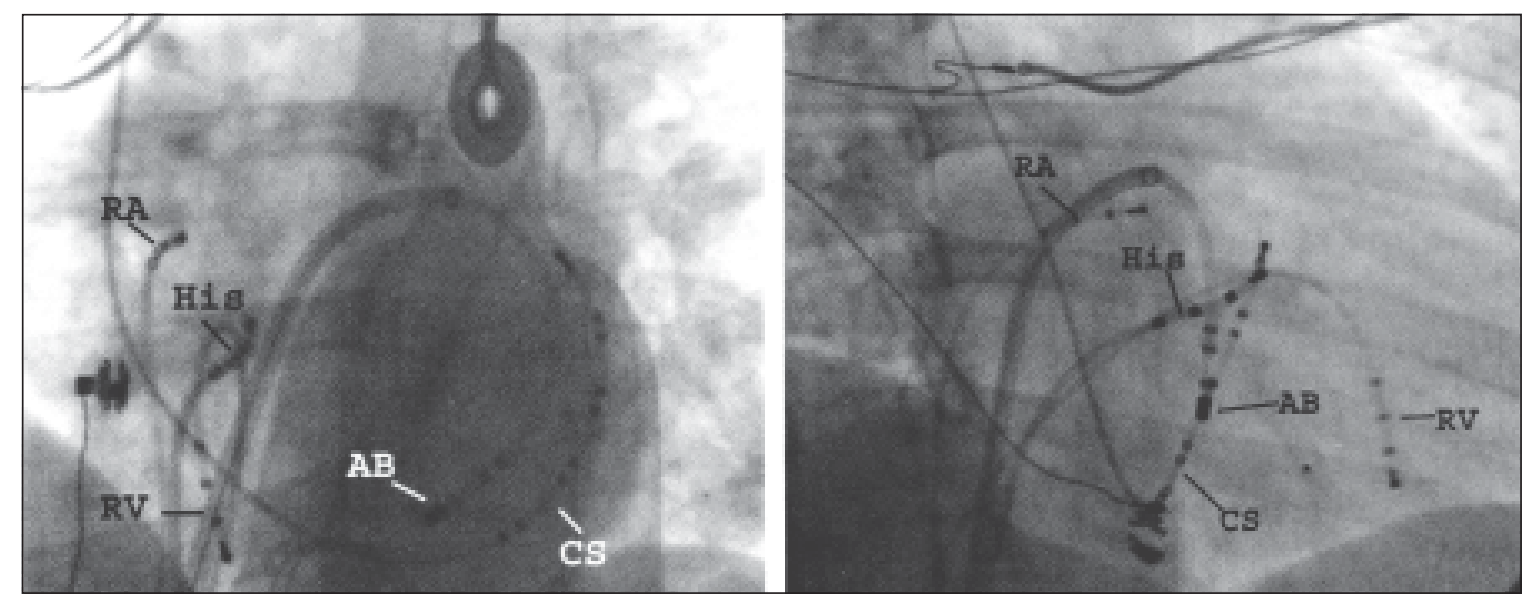

Fig.-2: Catheter positions in for transseptal approach to ablation of left free wall accessory pathway. A left anterior oblique view showing the preformed transseptal sheath crossing the foramen ovale and used to position the ablation catheter $(A B)$ along the proximal mitral annulus. B right anterior oblique view of catheter position. 
event of catheter displacement, unsuccessful blocking of Accessory pathway conduction in ten seconds or after sixty seconds of the effective $R F$ application. After twenty seconds of the effective application, we performed the programmed atrial and ventricular stimulation to confirm the absence of AP conduction. A test with adenosine (6 to 18 mg bolus IV) was carried out only in doubtful cases.

\section{Variables studied}

The following variavbles were analyzed and compared between the two groups: success rate, complications, ablation time (AT), puncture time (PT), fluoroscopy time (FT), total time of procedure (TT) and number of RF applications. We defined PT as the time spent in performing the arterial or TS puncture and AT as the time elapsed from the beginning of the mapping through the effective application. These variables were also analyzed according to the location of the AP (septal or free wall), the characteristics of the AP (overt or concealed).

\section{Statistical analysis}

Done by applying SPSS method, Fisher's exact test, Student's t testwas done as applicable. For all test we adopted the level of significance of $5 \%(p<0.05)$.

\section{Resutls:}

The patients of both groups had similar baseline characteristics (table I). Mean age of study population in TSA \& RAA groups where $35.60+$ 11.63 and $37.63+10.49$ years respectively. Among them $22(73.3 \%)$ were male and $8(26.7 \%)$ were female each group. Study also revealed that 13\% of patient in TSA group and 13\% patient of RAA group were diabetic, $33.3 \%$ and $23.3 \%$ patient were hypertensive, $10 \%$ and $7 \%$ patient had bronchial asthma, $3.3 \%$ and $2 \%$ patient had epilepsy respectively. All patient took similar antiarrahythmic drugs before ablation. Among them 16 and 18 patient took verapamil, 5 and 3 patient took Metaprolol, 6 and 7 patients took amiodarone, 3 and 2 patient. Sotaloli, 2 and 1 patient was suffering from Valvular heart disease, 1 and 1 patient form atrial septal defect in both TSA and RAA group respectively. Analysis revealed no statistically significant difference as regards to baseline Charecteristics. ( $>00.05)$.

Table II showed that electrocardiogram among the patients of TSA Group 13 (43.3\%) had SVT with WPW and 11 (36.7\%) had SVT without WPW, 6(20\%) atrial fibrillation with WPW and in RAA Group 11 (36.7\%) had SVT with WPW, 8 (26.7\%) had SVT without WPW, 11 (36.7\%) atrial fibrillation with WPW. There

Table I

Baseline characteristics of patients in both groups

\begin{tabular}{|c|c|c|c|}
\hline & \multicolumn{2}{|c|}{ Group } & \multirow[t]{2}{*}{ p value } \\
\hline & TSA n -30 & RAA n-30 & \\
\hline \multicolumn{4}{|l|}{ Age (in year) } \\
\hline Mean $\pm \mathrm{SD}$ & $35.60 \pm 11.63$ & $37.63 \pm 10.44$ & $0.479 *$ \\
\hline \multicolumn{4}{|l|}{ Sex } \\
\hline - Male & $22(73.3)^{\#}$ & $22(73.3)$ & $0.999 * *$ \\
\hline - Female & $8(26.7)$ & $8(26.7)$ & \\
\hline \multicolumn{4}{|l|}{ Concomitant Medical Illness } \\
\hline - $\mathrm{DM}$ & $4(13.3)$ & $4(13.3)$ & $0.999 * * *$ \\
\hline - $\mathrm{HTN}$ & $10(33.3)$ & $7(23.3)$ & $0.390 * *$ \\
\hline - Bronchial asthma & $3(10.0)$ & $2(6.7)$ & $0.999 * * *$ \\
\hline • $\quad$ Epilepsy & $1(3.3)$ & $2(6.7)$ & $0.999 * * *$ \\
\hline \multicolumn{4}{|c|}{ Antiarrhythmic Drugs used before ablation } \\
\hline - Verapamil & $16(53.3)$ & $18(60.0)$ & $0.827 * *$ \\
\hline - Metaprolol & $5(16.7)$ & $3(10.0)$ & \\
\hline - Amiodarone & $6(20.0)$ & $7(23.3)$ & \\
\hline - $\quad$ Sotalol & $3(10.0)$ & $2(6.7)$ & \\
\hline \multicolumn{4}{|l|}{ Structural Heart Disease } \\
\hline - Valvular heart disease & $2(6.7)$ & $1(3.3)$ & $0.999 * * *$ \\
\hline - Atrial Septal defect & $1(3.3)$ & $1(3.3)$ & $0.999 * * *$ \\
\hline
\end{tabular}


is no statistical significance of differences in electrocardiographic finding in both groups ( $p>0.05$ ).

Table III shows the locations of accessory's pathways in the left free wall. Among the TSA and RAA groups, WPW left-lateral pathway was present in $14(46 \%)$ and $10(33.3 \%)$, concealed left lateral in $11(36.7 \%)$ and $8(26.7 \%)$, WPW left posterior in $2(6.7 \%)$ and $11(36.7 \%)$, WPW anterolateral in 2 $(6.7 \%)$ and 0 , concealed left posterior in $1(3.3 \%)$ and $1(3.3 \%)$ respectively. There is no statistically significant difference in the locations of different accessory pathways in both groups. On the other hand manifest pathway was found in about $60 \%$ patients of TSA and 70\% of RAA group, left lateral accessory pathways were about $73 \%$ in TSA group and $60 \%$ in RAA group This table revealed that manifest and left lateral pathway is more common accessory pathways in both groups ( $>00.05)$.

Table IV The time spent for arterial punctures (PT) was shorter than for TS punctures $(p<0.0001)$. However, in the TSA group the AT was shorter ( $p$ $=0.006$ ) and the number of RF applications was smaller $(p=0.042)$. There was no significant statistical difference between the TSA and RAA groups as regards FT and TT (table 4).

\section{Success Rate}

Primary success in the ablation (first session) was achieved in $96.7 \%$ of the patients (29/30 patients) in the TSA group and in $80 \%$ (24/30 patients) in the RAA group $(p=0.103)$. Initial failure of transseptal method switched to retrograde method in one (1) Patient with successful ablations done in same session. But initial failure of retrograde approach in 6 patients successful ablation was done by transseptal approach in sepatate session. Crossover to transsptal method was done during a separate session to avoid complication related to access, with success, defining a total efficacy of $100 \%$ (figure 1).

\section{Complications}

There were no major complications but some minor complications observed(Table-V). Haematoma was observed only in 3 (10\%) patient of RAA group. Other complications include hypotension 1(3.3\%) and $2(6.7 \%)$ patient and vasovegal reaction $1(3.3 \%)$ \& 1(3.3\%) in TSA \& RAA group respectively. This indicates that haematoma occur more in RAA

Table II

Arrhythmia at presentation by groups

\begin{tabular}{lccc}
\hline ECG & \multicolumn{2}{c}{ Group } & p value* \\
\cline { 2 - 3 } & TSA & RAA & \\
\hline SVT with WPW & $13(43.3)$ & $11(36.7)$ & 0.348 \\
SVT without WPW & $11(36.7)$ & $8(26.7)$ & \\
Atrial fibrillation withWPW & $6(20.0)$ & $11(36.7)$ & \\
\hline Total & $30(100.0)$ & $30(100.0)$ & \\
\hline
\end{tabular}

*Chi-square test was done to measure the level of significance.

\#Figure within parentheses indicates in percentage.

Table III

Location of different pathways in both groups

\begin{tabular}{lccc}
\hline Pathway & \multicolumn{2}{c}{ Group } & p value* \\
\cline { 2 - 3 } & TSA n=30 & RAA n=30 & \\
\hline Manifest(WPW) & $14(46.7)$ & $10(33.3)$ & 0.292 \\
Left lateral & $2(6.7)$ & $11(36.7)$ & 0.080 \\
Left posterior & $2(6.7)$ & $0(.0)$ & \\
Left anterolateral & & & 0.999 \\
Concealed & $11(36.7)$ & $8(26.7)$ & \\
Concealed left lateral & $1(3.3)$ & $1(3.3)$ & \\
Concealed left posterior &
\end{tabular}

${ }^{*}$ Chi-square test was done to measure the level of significance.

\#Figure within parentheses indicates in percentage. 
Table IV

Parameters (mean $\pm S D$ ) of radiofrequency application in both the groups

\begin{tabular}{lccc}
\hline & \multicolumn{2}{c}{ Group } & p value* \\
\cline { 2 - 3 } & TSA n=30 & RAA n=30 & \\
\hline Puncture time (in min) & $11.53 \pm 4.31$ & $8.10 \pm 2.17$ & 0.001 \\
Ablation time (in min) & $30.17 \pm 9.78$ & $39.73 \pm 15.55$ & 0.006 \\
Fluoroscopy time (in min) & $19.27 \pm 7.53$ & $20.43 \pm 8.09$ & 0.565 \\
Total time of procedure (in min) & $105.67 \pm 37.96$ & $119.40 \pm 42.28$ & 0.191 \\
Number of radiofrequency application & $2.33 \pm 1.09$ & $3.17 \pm 1.90$ & 0.042 \\
\hline
\end{tabular}

${ }^{*}$ t test was done to measure the level of significance.

Data was shown as mean $\pm \mathrm{SD}$

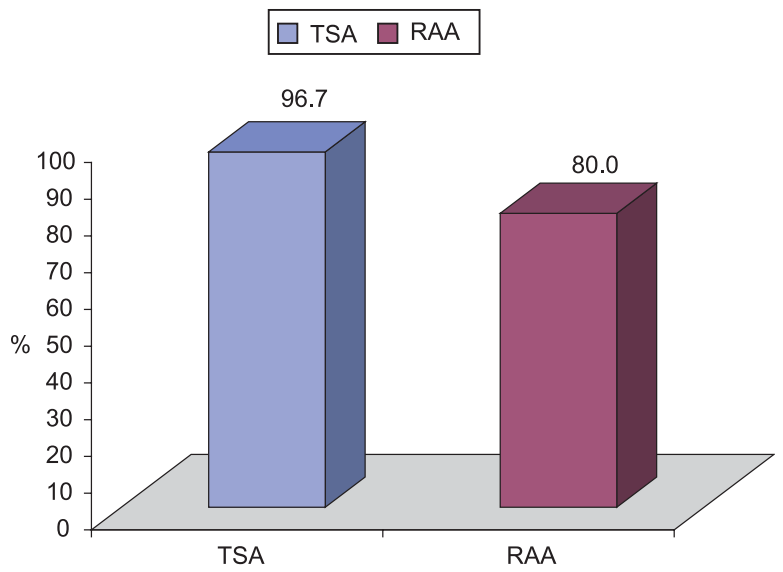

Fig.-3: Bar diagram of primary success rate of ablation of the patients

Table V

Distribution of complications by groups

\begin{tabular}{lccc}
\hline Complications & \multicolumn{2}{c}{ Group } & P value \\
\cline { 2 - 3 } & TSA(n=30) & RAA(n=30) & \\
\hline Major Complications & $0(.0)$ & $0(.0)$ & \\
Minor complications & & & 0.999 \\
- Haemorrhage & $1(3.3)$ & $0(.0)$ & 0.237 \\
- Haematoma & $0(.0)$ & $3(10.0)$ & 0.999 \\
- Hypotension & $1(3.3)$ & $2(6.7)$ & 0.999 \\
\hline
\end{tabular}

* Fisher's exact test was done to measure the level of significance.

\#Figure within parentheses indicates in percentage.

group, but overall rate of complication were same in both methods ( $p>0.05)$. No complications were observed which were directly related with the transeptal puncture.

\section{Discussion:}

In a set of 60 patients we prospectively compared the TSA technique with the conventional RAA used for catheter ablation of APs located in the left atrioventricular ring to test its efficacy and safety. The results showed that the TSA technique is as safe and effective as the RAA technique. This was also demonstrated that when used in a complementary fashion, they considerably increase the final success rate (100\%) final success rate.

Some studies compared both techniques in a retrospec-tive manner. Natale et $\mathrm{al}^{5}$ achieved a 
higher success rate in TSA ablation $(100 \%$ as compared to $88 \% ; \mathrm{p}<0.03)$ in a series with 80 patients. Lesh et al ${ }^{6}$ reported similar success rates (85\% as compared to $85 \%$; $\mathrm{p}=\mathrm{NS}$ ) for 106 patients, just like Manolis et al ${ }^{9}$ (86\% as compared to $87 \%$; $\mathrm{p}=\mathrm{NS}$ ) in 82 patients In Brazil, Sternick et al ${ }^{11}$ found a trend of better results with the TS approach (92\% as compared to $73 \% ; \mathrm{p}=0$.)

With the TS approach we had shorter ablation times $(p=0.01)$ and the number of applications was smaller ( $p=0.006)$; however, the fluoroscopy times (FT) and the total time, of procedure (TT) were similar. This may be explained by the longer time spent in the transeptal puncture (PT) compared with tha arterial puncture as was confirmed by the study $(\mathrm{p}<0.001)$ and as had been observed in a previous study. ${ }^{6}$ In the latter the authors reported a higher success rate with the TSA method in left anterolateral and posterolateral APs, but with no statistifal support, the comissures of the mitral valve in these locations supposedly make the ring less accessible through the ventricular surface according to the authors. ${ }^{6}$ Natale et al stated their preference for TSA for concealed APs since it is easier to map them in the atrial electrogram, when this technique is used, during tachycardia or ventricular stimulation.

In a more recent prospective and randomized study companing the RAA and TSA techniques and using only one catheter Katritsis et al observed shorter procedure time, smaller exposure to radiation and number of RF applications with the TSA technique.

In this study, when anlyzed, it was obseved that the benefits (shorter ablation time and smaller number of RF aplications) of the TSA technique were expressive only for APs located on free walls

Although it may be reached the Accessory pathways at atrial insertion using both techniques with high efficacy, ${ }^{8,10}$ the mapping of the Accessory pathways at ventricular insertion using the RAA technique was preferred since this approach provides greater stability to the catheter. In a subjective analysis, the operators found more difficulty in positioning the catheter via RAA in the anterolateral pathways.

With respect to children two studies stand out:",Vora et aliz obtained a $100 \%$ success rate in the ablation of left APs in their atrial insertions using the RA access in 36 patients and the TS access in thirteen. They reported a shorter FT for the RAA technique $(p=0.05) .{ }^{12}$ Law et al ${ }^{13}$ studied 136 children and obtained shorter FT, TT and smaller numbers of catheters used with the TSA technique, but without differenc relative to success rates and complications. ${ }^{13}$

Overall the studies suggest that the diffences found between the techniques may be related to the learning curve and to the experience of the operator, rather than to the specific technique used. ${ }^{12,13}$ When this study began ,the group was more experienced with procedures via RA, Even so the general trend was towards better results with the TSA approach. The transeptal puncture failed in only one patient. These results may be more expressive if a new study was carried out in the present, since the team now has a similar amount of experience with both approaches.

There is a consensus that the skill in the use of both techniques increases the final rate of success as compared to the use of each technique isolatedly. ${ }^{5,6,9,10}$ However, it is important to plan the procedure beforehand; Lesh et a1suggest that TS puncture should be avoided in patients during systemic anticoagulation (Initiated via RA), but it may be -performed-depending on the operator's prior experience and, if posslble, after the anticoagulation effect has been reversed.This is the strategy currently used in our laboratory in case of failure in the RA approach.

And lastly it is worth considering economic aspects when choosing the technique. The TSA procedure entails higher costs in connection with the specific material to be used. This factor, in combination with the technical difficulty associated with the TS puncture may limit its use on a routine basis.

Complications- Isolated cases of complications have been described for both techniques, thus preventing a statistical analysis. The most common are vascular complications in connection with the arterial puncture and pericardial effusion resulting from the puncture of the atrial wall during the TS puncture. ${ }^{910}$ Minich et al detected $30 \%$ new mild aortic regurgitation cases after procedures via RA. Kessler et al1 ${ }^{19}$ demonstrated the presence of leftto-right shunt through the interatrial septum following ablation via TS in six out of twelve patients. However, the clinical significance of these findings has not yet been determined.Formation 
of thrombus in the right atrium in the site of the transeptal puncture following left AP ablation has been recently reported in a patient. ${ }^{20}$ Greater complications were described by Calkins et $\mathrm{al}^{3}$ in six out of 158 patients submitted to the RA technique, including vascular complications, coronary thrombosis and aortic valve perforation Lesh et $\mathrm{l}^{6}$ reported a case of dissection of the left coronary artery during the RA technique followed by myocardial infarction It is speculated that $\mathrm{RF}$ lesions in the ventricular insertion of an AP in children may be arrhythmogenic, which however remains unproven to date. Saul et all ${ }^{8}$, reported the sudden death of a five-week old child two weeks after the ablation, a left Accessory pathway, which was attributed to ventricular arrhythmia. No major complication were observed in both groups in this study. Minor complications found in 3 patient of transseptal and 6 patients of retrograde method. Most of the complications were related to vascular access site haematoma and bleeding, which resolved spontaneously, leaving no sequelae.

\section{Conclusion:}

It is therefore concluded that catheter ablation of left Accessory pathways using the TSA technique is as effective as the conventional approach (RAA). When used in a complementary fashion (cross-over) they may increase the final success rate of ablation of left Accessory pathway, which suggests that mastery of both on the part of the electrophysialogist is important for his/ her daily practice. The TS approach was better than the RA approach as regards the ablation time and the number of RE applications, which may translate into less morbidity for the procedure. The absence of major complications confirms that both techniques are safe.

Conflict of Interest - None.

\section{References:}

1. Jackman WM, Xunzhang W; Friday KJ, Roman CR. Moulton KP, Beckman KJ, et al. Catheter ablation of accessory atrioventricular pathways (Wolff- ParkinsonWhite syndrome) by radiofrequency current. $N$ Eng $J$ Med 1991; 324: 1605-1611.

2. Lesh MD. Van Hare GF, Schamp DJ, Chien W, Lee MA, Griffin JC, et al. Curative percutaneous catheter ablation using radiofrequency energy for accessory pathways in all locations: results in 100 consecutive patients. J Am Coll Cardiol 1992; 19: 1303-1309,

3. Calkins H, Langberg J, Sousa J, el- Atassi R, Leon A, Kou W, et al. Radiofrequency catheter ablation of accessory atrioventricular connections in 250 patients: abbreviated therapeutic approach to Wollf-ParkinsonWhite Syndrome. Circulation 1992; 85: 1337-1349.

4. Calkins H, Kim Y-N, Schmaltz S, Sousa J, el- Atassi R, Leon A, et al. Eletrogram criteria for identification of appropriated target sites for radiofrequency catheter ablation of accessory atrloventricular connections. Circulation 1992; 85: 565-573

5. Natale A, Wathen M, Yee R, Wolfe K, Klein G. Atrial and ventricular approaches for rad iofrequency catheter ablation of left-sided accessory pathways. Am J Cardiol 1992; 70: 114-116.

6. Lesh MD, Van Hare GF, Scheinman MM, Ports TA, Epstein LA, Comparison of the retrograde and transseptal methods for ablation of left free wall accessory pathways. J Am Coll Cardiol 1993; 22: 542549 .

7. Swartr JF; Tracy CM, Fletcher RD. Radiofrequency endocardial catheter ablation of accessory atnoventncular pathway atrial insertion sites. Circulation 1993; 87: 487-899.

8. Saul JP, Hulse E, De W, Weber AT, Rhodes LA, Lock JE, et al. Catheter ablation of accessory atrioventricular pathway in young patients: use of long vascuiarsheats, the transseptal approach and a retrograde left posterior parallei approach. J Am Coll Cardiol 1993;21:571-583.

9. Manolis AS, Wang PJ, Mark Estes 3"' NA, Dhala AA, Blanck Zl Bajwa TK, et al. Radiofrequency ablation of left-sided accessory pathways: transaortic versus transseptal approach. Am Heart J 1994; 128: 896-902.

10. Deshpande SS, Bremner S, Sra JS. Ablation of left free-wall accessory pathways using radiofreyuency energy at the atrial insertion site: Transseptal versus transaortic approach. J Cardiovasc Electrophysiol. 1994; 5: 219-231.

11. Sternick EB, Gerken LM, Barbosa MR. Ablacao per caterer com radiofrequencia em vias acessoriasesquerdas: Abordagem transaortica versus transseptal. Reblampa 1997; 10: 177-185.

12. Vora AM, McMahon S, Jazayeri MR, Dhala AA. Ablation of atrial insertion sites of left-sided accessory pathways in children: efficacy and safety of transseptal versus transaortic approach. Pediatr Cardiol 1997; 18: 332338.

13. Law IH, Eischbach PS, Leroy S, Lloyd TR, Rocchini AP, Dick M 1 Ideas to left atrium for delivery of radiofrequency ablation in young patients: retrograde aortic vs transseptal approach. Pediatr Cardiol 2001; $22: 204-209$. 
14. Brockenbrough E, Braunwald E. A new technique for left ventricular angiocardiography and transseptal left heart catheterization. Am J Cardiol 1960 ; 6: 16021607.

15. 15. Mullins CE. Transseptal left heart catheterization: experience with a new technique in 520 pediatric and adult patients. Pediatr Cardiol 1983; 4: 239-46.

16. De Ponti R. Zardini M, Storti C, Longobardi M, SalernoUrlarte JA. Trans-septal catheterization tor radlofrequency catheter ablation of cardiac arrhythmias: results and safety of a simplified niethod. Eur Heart $J$ 1998; 19:943-50.

17. Katritsis D, Giagitzoglou E, Korovesis S, Zambartas C. Comparison of the transseptal approach to the transaortic approach for ablation of left-sided accessory pathways in patients with Wo!ff-Parkinson-White Syndrome. Am J Cardiol 2003; 91: 610-3.

18. Minich L, SniderA, Dick M II. Doppler detection of va1vular regurgitation after radioNquency ablation of accessory connections. Am J Cardiol 1992;70:116-8.

19. Kessler DJ, Pirwitz MJ, Horton RP, Canby RC, Welch PJ. Joglar JA. Intracardiac shunts resulting frorn transseptal catheterization tor ablation of accessory pathways in otherwise normal hearts. Arn J Cardiol 1998: 82: 391-2.

20. Bosquet S, Sunthorn H, Zaim S, Lerch R, Mach F Right atnal thrombus formation after radiofrequency catheter ablation of left-sided accessory pathway using a transseptal approach. Pacing Chin Electrophysiol 2002; 25: $1146-8$. 